\title{
Services et publications en ligne
}

L'IMCCE et le Bureau des longitudes proposent, sur leurs sites web respectifs, des services de calcul d'éphémérides et un choix de publications en rapport avec le contenu de cet ouvrage. Voici une liste de ces ressources et les adresses web pour y accéder.

\section{Services en ligne}

- Formulaires de calcul d'éphémérides (tout public) permettant d'obtenir des levers et couchers de planètes, de calculer des éphémérides de position ou pour l'observation physique des corps du Système solaire, d'obtenir les prédictions des éclipses de Lune et de Soleil ou des phénomènes de satellites des planètes.

u https://ssp.imcce.fr/forms

- Web services de calcul d'éphémérides (développeurs et public averti) permettant de développer des applications qui nécessitent d'accéder à des informations sur les corps du Système solaire (SsODNet), de calculer des éphémérides des corps du Système solaire (Miriade), de rechercher ces corps dans des images astronomiques (SkyBoT), ou de manipuler leurs spectres (M4AST).

[T https://ssp.imcce.fr/webservices/

\section{Éphémérides planétaires}

- INPOP, solution orbitale du Soleil, des planètes, de Pluton et de la Lune.

[ https://www.imcce.fr/inpop

- CALCEPH, librairie de calcul des éphémérides planétaires. 


\section{Publications institutionnelles}

- Connaissance des temps, ouvrage d'éphémérides destiné aux astronomes, enseignants et étudiants. Publication annuelle disponible aux formats papier ou ebook, accompagnée du logiciel eCDT de calcul d'éphémérides.

[T https://www . imcce.fr/publications/publications-institutionnelles/\#1

- Guide de données astronomiques (Annuaire du Bureau des longitudes), publié chaque année et répondant aux besoins de l'observateur.

¿ https://www.imcce.fr/publications/publications-institutionnelles/\#2

\section{Notes scientifiques et techniques}

- Publications des travaux de l'IMCCE liés aux recherches théoriques de mécanique céleste ou observationnelles en astrométrie, et aux travaux plus techniques appliqués dans l'élaboration des éphémérides.

c] https://www.imcce.fr/publications/publications-recherche/nst

\section{Publications du Bureau des longitudes}

- Ouvrages scientifiques et Cahiers des sciences de l'Univers succédant aux quatre volumes de l'Encyclopédie scientifique de l'Univers.

c https://www . bureau-des-longitudes.fr/publications.htm

\section{Ouvrages pour tous}

- Agenda astronomique, publication annuelle thématique fournissant de nombreuses informations destinées au grand public.

¿ https://www.imcce.fr/publications/ouvrages-pour-tous/\#1

- Divers ouvrages édités par l'IMCCE destinés à un large public.

七 https://Www.imcce.fr/publications/ouvrages-pour-tous/\#1 